\title{
On Addition Formulae for Sigma Functions of Telescopic Curves
}

\author{
Takanori AYANO ${ }^{\dagger}$ and Atsushi NAKAYASHIKI ${ }^{\ddagger}$ \\ $\dagger$ Department of Mathematics, Osaka University, Toyonaka, Osaka 560-0043, Japan \\ E-mail: tayano7150@gmail.com \\ $\ddagger$ Department of Mathematics, Tsuda College, Kodaira, Tokyo 187-8577, Japan \\ E-mail: atsushi@tsuda.ac.jp
}

Received March 13, 2013, in final form June 14, 2013; Published online June 19, 2013

http://dx.doi.org/10.3842/SIGMA.2013.046

\begin{abstract}
A telescopic curve is a certain algebraic curve defined by $m-1$ equations in the affine space of dimension $m$, which can be a hyperelliptic curve and an $(n, s)$ curve as a special case. We extend the addition formulae for sigma functions of $(n, s)$ curves to those of telescopic curves. The expression of the prime form in terms of the derivative of the sigma function is also given.
\end{abstract}

Key words: sigma function; tau function; Schur function; Riemann surface; telescopic curve; gap sequence

2010 Mathematics Subject Classification: 14H70; 37K20; 14H55; 14K25

\section{Introduction}

In this paper we study the multivariate sigma functions of telescopic curves and derive addition formulae together with their degenerate limits.

The multivariate sigma function originally introduced by F. Klein $[12,13]$ for hyperelliptic curves is generalized and extensively studied for the last decade (see [6] and references therein). Compared with Riemann's theta function, the sigma function is more algebraic and is directly related with the defining equation of an algebraic curve. A typical example where this nature of the sigma function is exhibited is the inversion problem of algebraic integrals. It is well known that the solution of Jacobi's inversion problem for a hyperelliptic curve has a simple description by hyperelliptic $\wp$-functions, the second logarithmic derivatives of the sigma function $[2,6]$. The inversion of hyperelliptic or more general algebraic integrals of genus $g$ on the Abel-Jacobi image $W_{k}$ of the $k$-th symmetric products of the curve with $k<g$ is extensively studied in connection with the problem of mathematical physics (see [3,6] and references therein). This problem is intimately related with the problem on the vanishing of the derivatives of the sigma function on $W_{k}$. Recently it is recognized that the approach from the view point of tau functions of integrable hierarchies provides a general and effective method to study such a problem [21].

In Sato's theory of Kadomtsev-Petviashvili (KP) hierarchy [24] the tau function is constructed from a point of the universal Grassmann manifold (UGM). For a solution corresponding to an algebraic curve the point of UGM is specified by expanding functions or sections of bundles on the curve using a local coordinate at a given point. The point of UGM obtained in this way belongs to the cell of UGM labeled by the partition $\lambda$ determined from the gap sequence at the point. The series expansion of the corresponding tau function begins from Schur function associated with $\lambda$. The tau function corresponding to the point of UGM specified by the affine ring of an $(n, s)$ curve [7] had been used to study the sigma function in [20, 21]. 
Therefore it is important to have such a pair $\left(X, p_{\infty}\right)$ of an algebraic curve $X$ and a point $p_{\infty} \in X$ that satisfies the following two conditions. The first is that a basis, as a vector space, of the space of regular functions on $X \backslash\left\{p_{\infty}\right\}$ can be explicitly described. The second is that the gap sequence at $p_{\infty}$ can be computable. A traditional example is a non-singular plane algebraic curve which can be completed by one point $\infty\left(=p_{\infty}\right)$ such as a hyperelliptic curve of odd degree or more generally an $(n, s)$ curve. Telescopic curves give new examples. They can be hyperelliptic and $(n, s)$ curves as special cases and are not realized as non-singular plane algebraic curves in general. Before explaining telescopic curves let us briefly explain the origin of the term "telescopic".

Let $a_{1}, \ldots, a_{m}$ be relatively prime positive integers. For a nonnegative integer $n$ the problem of determining nonnegative integers $x_{1}, \ldots, x_{m}$ satisfying the Diophantine equation

$$
n=a_{1} x_{1}+\cdots+a_{m} x_{m}
$$

has been studied in number theory since early times. It is well known that the equation (1) has a solution if $n$ is sufficiently large. The greatest number $n$ for which the equation (1) has no solution is called Frobenius number and we denote it by $F\left(a_{1}, \ldots, a_{m}\right)$. Brauer [4] gave an upper bound of the Frobenius number as

$$
F\left(a_{1}, \ldots, a_{m}\right) \leq-a_{1}+\sum_{i=2}^{m} a_{i}\left(\frac{d_{i-1}}{d_{i}}-1\right),
$$

where $d_{i}=\operatorname{gcd}\left(a_{1}, \ldots, a_{i}\right)$. Also Brauer [4] and Brauer and Seelbinder [5] showed that the equality in (2) holds if and only if

$$
\frac{a_{i}}{d_{i}} \in \frac{a_{1}}{d_{i-1}} \mathbb{Z}_{\geq 0}+\cdots+\frac{a_{i-1}}{d_{i-1}} \mathbb{Z}_{\geq 0}, \quad 2 \leq i \leq m .
$$

The condition (3) is introduced in Brauer [4] for the first time and now it is called telescopic condition. For $a_{1}, \ldots, a_{m}$ satisfying (3) the semigroup $S:=a_{1} \mathbb{Z}_{\geq 0}+\cdots+a_{m} \mathbb{Z}_{\geq 0}$ is called telescopic semigroup. The telescopic semigroup has many nice structures and has many applications in algebraic geometric code, algebraic curve cryptography, and commutative algebra (see for instance $[11,17,18])$.

In [18] Miura introduced a certain canonical form, Miura canonical form, for defining equations of any non-singular algebraic curve. A telescopic curve [18] is a special curve for which Miura canonical form is easy to determine. Let $m \geq 2$ and $\left(a_{1}, \ldots, a_{m}\right)$ a sequence of relatively prime positive integers satisfying the telescopic condition (3). Then the telescopic curve associated with $\left(a_{1}, \ldots, a_{m}\right)$ or the $\left(a_{1}, \ldots, a_{m}\right)$ curve is the algebraic curve defined by certain $m-1$ equations in $\mathbb{C}^{m}$. The form of defining equations is explicitly computable from $\left(a_{1}, \ldots, a_{m}\right)$ (see (5)). If a telescopic curve is non-singular, then it can be completed by adding one point, say $\infty$, and the gap sequence at $\infty$ becomes the complement of the telescopic semigroup [1, 18]. In such a case the genus of the curve is also explicitly computable (see (6)).

In [21] the vanishing and the expansion of the sigma function of an $(n, s)$ curve on the Abel-Jacobi image $W_{k}$ for $k<g$ are studied using the properties of the tau function of the KP-hierarchy. Those results are then applied to study the restriction of the addition formulae on $W_{k}$ to the lower strata $W_{k^{\prime}}$ with $k^{\prime}<k$. On the other hand the sigma function of the telescopic curve is explicitly constructed in [1]. In this paper we show that almost results in [21] are extended to the case of telescopic curves. The results imply two things. The first is that telescopic curves are natural objects to study sigma functions. We expect, more generally, the Miura canonical form is suitable to describe properties of the sigma functions. The second is that the tau function approach is effective in a more general case than that of $(n, s)$ curves. 
We expect that the method by integrable hierarchies can equally be efficient to study sigma functions with characteristics of arbitrary Riemann surfaces [15].

Finally we comment that the sigma functions of certain space curves, which are not telescopic, are studied in $[14,16]$.

The present paper is organized as follows. In Section 2 the definition and examples of telescopic curves are given. The construction of the sigma function, up to the normalization constant, associated with telescopic curves is reviewed in Section 3. The local coordinate $z$ at $\infty$ is specified and the expression in terms of $z$ of the variables appearing in the defining equations of the curve is given. This is necessary to determine constants appearing in every formula in later sections. In Section 4 the expression of the tau function is given using the sigma function. The normalization constant necessary in the definition of the sigma function is specified with the help of it. In Section 5 main results including the addition formulae are given. Their proofs are indicated in Section 6. The example of addition formulae is given for a $(4,6,5)$ curve in Section 7. In Appendix A the detailed properties on the series expansion of the sigma function are given for the sake of completeness of the construction of the sigma function.

\section{Telescopic curves}

In this section we briefly review the definition and properties of telescopic curves following $[1,18]$ and give some examples.

Let $m \geq 2,\left(a_{1}, \ldots, a_{m}\right)$ a sequence of positive integers such that $\operatorname{gcd}\left(a_{1}, \ldots, a_{m}\right)=1$ and $d_{i}=\operatorname{gcd}\left(a_{1}, \ldots, a_{i}\right)$ for $1 \leq i \leq m$. We call $\left(a_{1}, \ldots, a_{m}\right)$ telescopic if

$$
\frac{a_{i}}{d_{i}} \in \frac{a_{1}}{d_{i-1}} \mathbb{Z}_{\geq 0}+\cdots+\frac{a_{i-1}}{d_{i-1}} \mathbb{Z}_{\geq 0}, \quad 2 \leq i \leq m .
$$

The following examples of telescopic sequences are given in [22].

\section{Example 1.}

(i) $\left(a_{1}, a_{2}\right)$, s.t. $\operatorname{gcd}\left(a_{1}, a_{2}\right)=1$.

(ii) $(k, k+2, k+1)$, s.t. $k$ even.

(iii) $(a b, b c, a+c)$, s.t. $\operatorname{gcd}(a, c)=1, \operatorname{gcd}(b, a+c)=1$.

(iv) $\left(a_{1}, \ldots, a_{m}\right)$, s.t. $a_{i}=a^{m-i} b^{i-1}, a>b, \operatorname{gcd}(a, b)=1$.

Notice that whether a sequence is telescopic depends on the order of the numbers. For example, $(4,6,5)$ is telescopic while $(4,5,6)$ is not.

In the following we assume that $A_{m}=\left(a_{1}, \ldots, a_{m}\right)$ is telescopic unless otherwise stated.

Let

$$
B\left(A_{m}\right)=\left\{\left(l_{1}, \ldots, l_{m}\right) \in \mathbb{Z}_{\geq 0}^{m} \mid 0 \leq l_{i} \leq \frac{d_{i-1}}{d_{i}}-1 \text { for } 2 \leq i \leq m\right\} .
$$

Lemma 1 ([1, 18]). For any $a \in a_{1} \mathbb{Z}_{\geq 0}+\cdots+a_{m} \mathbb{Z}_{\geq 0}$, there exists a unique element $\left(k_{1}, \ldots, k_{m}\right)$ of $B\left(A_{m}\right)$ such that

$$
\sum_{i=1}^{m} a_{i} k_{i}=a .
$$

By this lemma, for any $2 \leq i \leq m$, there exists a unique sequence $\left(l_{i 1}, \ldots, l_{i m}\right) \in B\left(A_{m}\right)$ satisfying

$$
\sum_{j=1}^{m} a_{j} l_{i j}=a_{i} \frac{d_{i-1}}{d_{i}} .
$$


Consider $m-1$ polynomials in $m$ variables $x_{1}, \ldots, x_{m}$ given by

$$
F_{i}(x)=x_{i}^{d_{i-1} / d_{i}}-\prod_{j=1}^{m} x_{j}^{l_{i j}}-\sum \kappa_{j_{1} \ldots j_{m}}^{(i)} x_{1}^{j_{1}} \cdots x_{m}^{j_{m}}, \quad 2 \leq i \leq m,
$$

where $\kappa_{j_{1} \ldots j_{m}}^{(i)} \in \mathbb{C}$ and the sum of the right hand side is over all $\left(j_{1}, \ldots, j_{m}\right) \in B\left(A_{m}\right)$ such that

$$
\sum_{k=1}^{m} a_{k} j_{k}<a_{i} \frac{d_{i-1}}{d_{i}}
$$

Let $X^{\text {aff }}$ be the common zeros of $F_{2}, \ldots, F_{m}$ :

$$
X^{\mathrm{aff}}=\left\{\left(x_{1}, \ldots, x_{m}\right) \in \mathbb{C}^{m} \mid F_{i}\left(x_{1}, \ldots, x_{m}\right)=0,2 \leq i \leq m\right\} .
$$

In $[1,18] X^{\text {aff }}$ is proved to be an affine algebraic curve. We assume that $X^{\text {aff }}$ is nonsingular. Let $X$ be the compact Riemann surface corresponding to $X^{\text {aff }}$. Then $X$ is obtained from $X^{\text {aff }}$ by adding one point, say $\infty[1,18]$. It is proved in $[1,18]$ that $x_{i}$ has a pole of order $a_{i}$ at $\infty$. The genus of $X$ is given by $[1,18]$

$$
g=\frac{1}{2}\left(1+\sum_{i=2}^{m} a_{i} \frac{d_{i-1}}{d_{i}}-\sum_{i=1}^{m} a_{i}\right) .
$$

We call $X$ the $\left(a_{1}, \ldots, a_{m}\right)$ curve or the telescopic curve associated with $\left(a_{1}, \ldots, a_{m}\right)$. The numbers $a_{1}, \ldots, a_{m}$ are a generator of the semigroup of non-gaps at $\infty$.

\section{Example 2.}

(i) The telescopic curve associated with a pair of relatively prime integers $(n, s)$ is the $(n, s)$ curve introduced in [7].

(ii) For $A_{3}=(2 k, 2 k+2,2 k+1), k \geq 2$, in (ii) of Example 1, polynomials $F_{i}$ are given by

$$
\begin{aligned}
& F_{2}(x)=x_{2}^{k}-x_{1}^{k+1}-\sum^{(2)} \kappa_{i_{1}, i_{2}, i_{3}}^{(2)} x_{1}^{i_{1}} x_{2}^{i_{2}} x_{3}^{i_{3}}, \\
& F_{3}(x)=x_{3}^{2}-x_{1} x_{2}-\sum^{(3)} \kappa_{i_{1}, i_{2}, i_{3}}^{(3)} x_{1}^{i_{1}} x_{2}^{i_{2}} x_{3}^{i_{3}},
\end{aligned}
$$

where $\sum^{(i)}, i=2,3$ signify the sum over all $\left(i_{1}, i_{2}, i_{3}\right) \in B\left(A_{3}\right)$ such that

$$
2 k i_{1}+2(k+1) i_{2}+(2 k+1) i_{3}< \begin{cases}2 k(k+1) & \text { for } \sum^{(2)}, \\ 2(2 k+1) & \text { for } \sum^{(3)} .\end{cases}
$$

The genus of $X$ is $g=k^{2}$.

(iii) For $A_{3}=(a b, b c, a+c), a \neq 1$, in (iii) of Example 1, we have

$$
\begin{aligned}
& F_{2}(x)=x_{2}^{a}-x_{1}^{c}-\sum^{(2)} \kappa_{i_{1}, i_{2}, i_{3}}^{(2)} x_{1}^{i_{1}} x_{2}^{i_{2}} x_{3}^{i_{3}}, \\
& F_{3}(x)=x_{3}^{b}-x_{1} x_{2}-\sum^{(3)} \kappa_{i_{1}, i_{2}, i_{3}}^{(3)} x_{1}^{i_{1}} x_{2}^{i_{2}} x_{3}^{i_{3}},
\end{aligned}
$$

where $\sum^{(i)}, i=2,3$ denote the sum over all $\left(i_{1}, i_{2}, i_{3}\right) \in B\left(A_{3}\right)$ such that

$$
a b i_{1}+b c i_{2}+(a+c) i_{3}< \begin{cases}a b c & \text { for } \sum^{(2)} \\ b(a+c) & \text { for } \sum^{(3)}\end{cases}
$$

The genus of $X$ is

$$
g=\frac{1+a b c-a-c}{2} .
$$


(iv) For $A_{m}=\left(a_{1}, \ldots, a_{m}\right)$ in $(i v)$ of Example 1, we have

$$
F_{i}(x)=x_{i}^{a}-x_{i-1}^{b}-\sum_{a_{1} j_{1}+\cdots+a_{m} j_{m}<a a_{i}} \kappa_{j_{1} \ldots j_{m}}^{(i)} x_{1}^{j_{1}} \cdots x_{m}^{j_{m}}
$$

The genus of $X$ is

$$
g=\frac{a-b+(b-1) a^{m}-(a-1) b^{m}}{2(a-b)} .
$$

\section{Sigma function of telescopic curves}

An algebraic bilinear differential of a telescopic curve associated with $\left(a_{1}, \ldots, a_{m}\right)$ is explicitly constructed in [1]. Consequently an expression of the sigma function in terms of Riemann's theta function and some algebraic data had been given. We recall the results of [1] and add some necessary results for our purpose.

Let $X$ be a telescopic curve of genus $g \geq 1$ associated with $\left(a_{1}, \ldots, a_{m}\right)$ and $\left(l_{i 1}, \ldots, l_{i m}\right)$ the element of $B\left(A_{m}\right)$ specified by (4).

Lemma 2. For any $i$ we have $l_{i j}=0$ for $j \geq i$.

Proof. Since $A_{m}$ is telescopic, there exist $k_{1}, \ldots, k_{i-1} \in \mathbb{Z}_{\geq 0}$ such that

$$
a_{i} \frac{d_{i-1}}{d_{i}}=a_{1} k_{1}+\cdots+a_{i-1} k_{i-1}
$$

We prove that we can take $0 \leq k_{j}<d_{j-1} / d_{j}$ for any $j \geq 2$ by changing $k_{j}$ appropriately if necessary.

Suppose that $k_{j^{\prime}} \geq d_{j^{\prime}-1} / d_{j^{\prime}}$ for some $j^{\prime}$. Take the largest number $j$ satisfying this condition. Let us write

$$
k_{j}=\frac{d_{j-1}}{d_{j}} q+r
$$

with $q \geq 1,0 \leq r<d_{j-1} / d_{j}$. Since $A_{m}$ is telescopic, there exist $u_{1}, \ldots, u_{j-1} \in \mathbb{Z}_{\geq 0}$ such that

$$
a_{j} \frac{d_{j-1}}{d_{j}}=a_{1} u_{1}+\cdots+a_{j-1} u_{j-1} .
$$

Then we have

$$
a_{j} k_{j}=a_{j} \frac{d_{j-1}}{d_{j}} q+a_{j} r=a_{1} q u_{1}+\cdots+a_{j-1} q u_{j-1}+a_{j} r .
$$

Substituting this into (7) we get the expression of the form (7) with $0 \leq k_{l}<d_{l-1} / d_{l}$ for any $l \geq j$. Repeating similar change of $k_{j^{\prime}}$ for $j^{\prime}$ smaller than $j$ successively we finally get the expression of $a_{i} d_{i-1} / d_{i}$ of the form (7) with $0 \leq k_{j}<d_{j-1} / d_{j}$ for any $j \geq 2$.

By the definition of $B\left(A_{m}\right),\left(k_{1}, \ldots, k_{i-1}, 0, \ldots, 0\right) \in B\left(A_{m}\right)$. Since the element of $B\left(A_{m}\right)$ satisfying (4) is unique by Lemma $1,\left(l_{i 1}, \ldots, l_{i m}\right)=\left(k_{1}, \ldots, k_{i-1}, 0, \ldots, 0\right)$.

For the defining equations (5), we assign degrees as

$$
\operatorname{deg} \kappa_{j_{1} \ldots j_{m}}^{(i)}=a_{i} d_{i-1} / d_{i}-\sum_{k=1}^{m} a_{k} j_{k} .
$$


Lemma 3. It is possible to take a local parameter $z$ around $\infty$ such that

$$
x_{1}=\frac{1}{z^{a_{1}}}, \quad x_{k}=\frac{1}{z^{a_{k}}}\left(1+\sum_{l=1}^{\infty} e_{k l} z^{l}\right), \quad 2 \leq k \leq m,
$$

where $e_{k l}$ belongs to $\mathbb{Q}\left[\left\{\kappa_{j_{1} \ldots j_{m}}^{(i)}\right\}\right]$ and is homogeneous of degree $l$ if $e_{k l} \neq 0$.

Proof. It is possible to take a local parameter $z_{0}$ around $\infty$ such that

$$
x_{1}=\frac{1}{z_{0}^{a_{1}}}
$$

Let $\zeta=\exp \left(2 \pi \sqrt{-1} / a_{1}\right)$ and $i \geq 0$. Then $z_{i}:=\zeta^{i} z_{0}$ is also a local parameter around $\infty$. Let $e_{k}^{(i)}$ be the coefficient of the first term of the series expansion of $x_{k}$ around $\infty$ with respect to $z_{i}$ :

$$
x_{k}=\frac{e_{k}^{(i)}}{z_{i}^{a_{k}}}\left(1+O\left(z_{i}\right)\right), \quad 2 \leq k \leq m .
$$

We prove that there exists $i$ such that $e_{2}^{(i)}=\cdots=e_{m}^{(i)}=1$.

Let $e^{(i)}=\left(e_{2}^{(i)}, \ldots, e_{m}^{(i)}\right)$ for $0 \leq i<a_{1}$. First we show $e^{(i)} \neq e^{(j)}$ for $i \neq j$. Suppose $e^{(i)}=e^{(j)}$. Since $e_{k}^{(i)}=\zeta^{a_{k} i} e_{k}^{(0)}$, we have $\zeta^{a_{k}(i-j)}=1$ for $k=2, \ldots, m$. From $\operatorname{gcd}\left(a_{1}, \ldots, a_{m}\right)=1$ and $0 \leq i, j<a_{1}$, we have $i=j$.

By Lemma 2 the defining equations of $X$ are as follows:

$$
x_{k}^{d_{k-1} / d_{k}}=x_{1}^{l_{k 1}} \cdots x_{k-1}^{l_{k k-1}}+\sum \kappa_{j_{1} \ldots j_{m}}^{(k)} x_{1}^{j_{1}} \cdots x_{m}^{j_{m}}, \quad 2 \leq k \leq m .
$$

By substituting (9) to (10) and comparing the coefficients of $z_{i}^{-a_{k} d_{k-1} / d_{k}}$, we have

$$
\left(e_{2}^{(i)}\right)^{d_{1} / d_{2}}=1, \quad\left(e_{k}^{(i)}\right)^{d_{k-1} / d_{k}}=\left(e_{2}^{(i)}\right)^{l_{k 2}} \cdots\left(e_{k-1}^{(i)}\right)^{l_{k k-1}}, \quad 3 \leq k \leq m .
$$

Let

$$
S=\left\{\left(s_{2}, \ldots, s_{m}\right) \in \mathbb{C}^{m-1} \mid s_{2}^{d_{1} / d_{2}}=1, s_{k}^{d_{k-1} / d_{k}}=s_{2}^{l_{k 2}} \cdots s_{k-1}^{l_{k k-1}}, 3 \leq k \leq m\right\} .
$$

Since $\sharp S=\left(d_{1} / d_{2}\right) \cdots\left(d_{m-1} / d_{m}\right)=\left(d_{1} / d_{m}\right)=a_{1}$ and $e^{(i)} \in S$ for $i=0, \ldots, a_{1}-1$, we have

$$
S=\left\{e^{(0)}, \ldots, e^{\left(a_{1}-1\right)}\right\} \text {. }
$$

Since $(1, \ldots, 1) \in S$, there exists $i$ such that $e^{(i)}=(1, \ldots, 1)$. For $z:=z_{i}, x_{k}$ is expanded as

$$
x_{1}=\frac{1}{z^{a_{1}}}, \quad x_{k}=\frac{1}{z^{a_{k}}}\left(1+\sum_{l=1}^{\infty} e_{k l} z^{l}\right), \quad e_{k l} \in \mathbb{C} .
$$

Let us prove that $e_{k l}$ belongs to $\mathbb{Q}\left[\left\{\kappa_{j_{1} \ldots j_{m}}^{(i)}\right\}\right]$ and is homogeneous of degree $l$ if $e_{k l} \neq 0$. We define the order $<$ in the set $\left\{e_{k l}\right\}$ so that $e_{k^{\prime} l^{\prime}}<e_{k l}$ if

1) $l^{\prime}<l$ or

2) $l^{\prime}=l$ and $k^{\prime}<k$. 
We prove the statement by induction on this order. By (5) and Lemma 2 we have

$$
\begin{aligned}
\left(1+\sum_{j=1}^{\infty} e_{k j} z^{j}\right)^{\frac{d_{k-1}}{d_{k}}}= & \prod_{s=2}^{k-1}\left(1+\sum_{j=1}^{\infty} e_{s j} z^{j}\right)^{l_{k s}} \\
& +\sum \kappa_{j_{1} \ldots j_{m}}^{(k)} z^{\frac{a_{k} d_{k-1}}{d_{k}}-\sum_{s=1}^{m} a_{s} j_{s}} \prod_{s=2}^{m}\left(1+\sum_{j=1}^{\infty} e_{s j} z^{j}\right)^{j_{s}},
\end{aligned}
$$

where we define the empty product from $s=2$ to 1 to be one in the first term of the right hand side.

In (11) for $k=2$, the coefficient of $z$ of the left hand side is $\left(d_{1} / d_{2}\right) e_{21}$ and that of the right hand side is the sum of $\kappa_{j_{1} \ldots j_{m}}^{(2)}$ with $\left(j_{1}, \ldots, j_{m}\right)$ satisfying the equation $\left(a_{2} d_{1} / d_{2}\right)-\sum_{s=1}^{m} a_{s} j_{s}=1$. Therefore the statement is valid for the minimal element $e_{21}$.

Assume that the statement holds for any $e_{k^{\prime} l^{\prime}}$ satisfying $e_{k^{\prime} l^{\prime}}<e_{k l}$. The coefficient of $z^{l}$ of the left hand side of (11) is $\left(d_{k-1} / d_{k}\right) e_{k l}+T$, where $T$ is a sum of $\prod_{i} e_{k q_{i}}$ satisfying $\sum_{i} q_{i}=l$ and $q_{i}<l$. In the right hand side of (11), the coefficient of $z^{l}$ of the first term is the sum of $\prod_{i} e_{p_{i} q_{i}}$ satisfying $2 \leq p_{i}<k$ and $\sum_{i} q_{i}=l$, and that of the second term is the sum of $\kappa_{j_{1} \ldots j_{m}}^{(k)} \prod_{i} e_{p_{i} q_{i}}$ with $\left(j_{1}, \ldots, j_{m}\right)$ satisfying $\sum_{i} q_{i}=l-\left(a_{k} d_{k-1} / d_{k}\right)+\sum_{s=1}^{m} a_{s} j_{s}$. Therefore, by the assumption of induction, we find that $e_{k l}$ belongs to $\mathbb{Q}\left[\left\{\kappa_{j_{1} \ldots j_{m}}^{(i)}\right\}\right]$ and is homogeneous of degree $l$ if $e_{k l} \neq 0$.

For a meromorphic function $f$ on $X$ we denote by $\operatorname{ord}_{\infty}(f)$ the order of a pole at $\infty$. Then we have $\operatorname{ord}_{\infty}\left(x_{i}\right)=a_{i}$. We enumerate the monomials $x_{1}^{\alpha_{1}} \cdots x_{m}^{\alpha_{m}},\left(\alpha_{1}, \ldots, \alpha_{m}\right) \in B\left(A_{m}\right)$ according as the order of a pole at $\infty$ and denote them by $\varphi_{i}, i \geq 1$. In particular we have $\varphi_{1}=1$.

Let $\left(w_{1}, \ldots, w_{g}\right)$ be the gap sequence at $\infty$ :

$$
\left\{w_{i} \mid 1 \leq i \leq g\right\}=\mathbb{Z}_{\geq 0} \backslash\left\{\sum_{i=1}^{m} a_{i} \mathbb{Z}_{\geq 0}\right\}, \quad w_{1}<\cdots<w_{g} .
$$

In particular $w_{1}=1$, since $g \geq 1$.

A basis of holomorphic one forms is given by

$$
d u_{w_{i}}=-\frac{\varphi_{g+1-i}}{\operatorname{det} G(x)} d x_{1},
$$

where $G(x)$ is the Jacobian matrix

$$
G(x)=\left(\frac{\partial F_{i}}{\partial x_{j}}\right)_{2 \leq i, j \leq m} .
$$

The following lemma is proved in [1].

Lemma 4. We have $w_{g}=2 g-1$. In particular $d u_{2 g-1}$ has a zero of order $2 g-2$ at $\infty$.

More precisely we have the following properties.

\section{Proposition 1.}

(i) The following expansion is valid around $\infty$ :

$$
d u_{2 g-1}=z^{2 g-2}\left(1+\sum_{l=1}^{\infty} e_{l}^{\prime} z^{l}\right) d z
$$

where $e_{l}^{\prime}$ belongs to $\mathbb{Q}\left[\left\{\kappa_{j_{1} \ldots j_{m}}^{(i)}\right\}\right]$ and is homogeneous of degree $l$ if $e_{l}^{\prime} \neq 0$. 
(ii) For $1 \leq i \leq g$ the expansion of $d u_{w_{i}}$ at $\infty$ is of the form

$$
d u_{w_{i}}=z^{w_{i}-1}(1+O(z)) d z .
$$

Proof. (i) From Lemmas 2, 3, we have, around $\infty$,

$$
\operatorname{det} G(x)=a_{1} z^{-\sum_{i=2}^{m}\left(\left(d_{i-1} / d_{i}\right)-1\right) a_{i}}\left(1+\sum_{l=1}^{\infty} e_{l}^{\prime \prime} z^{l}\right) d z
$$

where $e_{l}^{\prime \prime}$ belongs to $\mathbb{Q}\left[\left\{\kappa_{j_{1} \ldots j_{m}}^{(i)}\right\}\right]$ and is homogeneous of degree $l$ if $e_{l}^{\prime \prime} \neq 0$. Therefore, from (6), we obtain the assertion.

(ii) Let $w_{i}^{*}=\operatorname{ord}_{\infty}\left(\varphi_{i}\right), W=\left\{w_{1}, \ldots, w_{g}\right\}$, and $W^{\prime}=\left\{w_{1}^{*}, \ldots, w_{g}^{*}\right\}$. Note that $W \cup W^{\prime}=$ $\{0,1, \ldots, 2 g-1\}$. If $w \in W^{\prime}$, then $w_{g}-w \in W$. In fact, if $w_{g}-w \in W^{\prime}$, then $w_{g} \in W^{\prime}$, which is contradiction. Since $w_{g}=2 g-1$, we have $2 g-1-w_{g+1-i}^{*}=w_{i}$ for any $i$. Therefore, from (12), Lemma 3, and Proposition 1(i), we obtain the assertion.

The algebraic bilinear differential takes the form

$$
\widehat{\omega}(x, y)=d_{y} \Omega(x, y)+\sum_{i=1}^{g} d u_{w_{i}}(x) d r_{i}(y),
$$

where $x=\left(x_{1}, \ldots, x_{m}\right), y=\left(y_{1}, \ldots, y_{m}\right)$ are points on $X$,

$$
\Omega(x, y)=\frac{\operatorname{det} H(x, y)}{\left(x_{1}-y_{1}\right) \operatorname{det} G(x)} d x_{1}
$$

$H=\left(h_{i j}\right)_{2 \leq i, j \leq m}$ with

$$
h_{i j}=\frac{F_{i}\left(y_{1}, \ldots, y_{j-1}, x_{j}, x_{j+1}, \ldots, x_{m}\right)-F_{i}\left(y_{1}, \ldots, y_{j-1}, y_{j}, x_{j+1}, \ldots, x_{m}\right)}{x_{j}-y_{j}},
$$

and $d r_{i}$ is a second kind differential with a pole only at $\infty$. By construction $\left\{d u_{w_{i}}, d r_{i}\right\}$ becomes a symplectic basis of the cohomology group $H^{1}(X, \mathbb{C})$ (see $\left.[1,19]\right)$.

Take a symplectic basis $\left\{\alpha_{i}, \beta_{i}\right\}$ of the homology group and define the period matrices by

$$
\begin{array}{ll}
2 \omega_{1}=\left(\int_{\alpha_{j}} d u_{w_{i}}\right), & 2 \omega_{2}=\left(\int_{\beta_{j}} d u_{w_{i}}\right), \\
-2 \eta_{1}=\left(\int_{\alpha_{j}} d r_{i}\right), & -2 \eta_{2}=\left(\int_{\beta_{j}} d r_{i}\right) .
\end{array}
$$

The normalized period matrix is given by $\tau=\omega_{1}^{-1} \omega_{2}$.

Let $\delta=\tau \delta^{\prime}+\delta^{\prime \prime}, \delta^{\prime}, \delta^{\prime \prime} \in \mathbb{R}^{g}$ be the Riemann's constant with respect to the choice $\left(\left\{\alpha_{i}, \beta_{i}\right\}, \infty\right)$. We set $\delta={ }^{t}\left({ }^{t} \delta^{\prime},{ }^{t} \delta^{\prime \prime}\right)$. Since $d u_{2 g-1}$ has a zero of order $2 g-2$ at $\infty$ by Lemma 4 , we have $\delta \in(\mathbb{Z} / 2)^{2 g}$.

We define the function $\widehat{\sigma}(u), u=\left(u_{w_{1}}, \ldots, u_{w_{g}}\right)$ by

$$
\widehat{\sigma}(u)=\exp \left(\frac{1}{2} t u \eta_{1} \omega_{1}^{-1} u\right) \theta[\delta]\left(\left(2 \omega_{1}\right)^{-1} u, \tau\right)
$$

where $\theta[\delta](u)$ is the Riemann's theta function with the characteristic $\delta$. 


\section{Relation with tau function}

In the case of $(n, s)$ curves the expression of the tau function of the KP-hierarchy in terms of the sigma function is given in $[8,10,20]$. For the tau function corresponding to the point of Sato's universal Grassmann manifold (UGM) specified by the affine ring of a telescopic curve a similar expression holds.

Let $A$ be the affine ring of $X$,

$$
A=\mathbb{C}\left[x_{1}, \ldots, x_{m}\right] / I,
$$

where $I$ is the ideal generated by $F_{i}(x), 2 \leq i \leq m$. As a vector space $A=\oplus_{i=1}^{\infty} \mathbb{C} \varphi_{i}$.

We embed $A$ in UGM as in Section 5 of [20] using the local parameter $z$ in (8) and denote $U^{A}$ the image of it. Let $\xi^{A}$ be the normalized frame of $U^{A}$ and $\tau\left(t ; \xi^{A}\right)$ the tau function corresponding to $\xi^{A}$. Then we have the expansion of the form

$$
\tau\left(t ; \xi^{A}\right)=s_{\lambda}(t)+\sum_{\lambda<\mu} \xi_{\mu} s_{\mu}(t)
$$

where $\lambda=\left(\lambda_{1}, \ldots, \lambda_{g}\right)$ is the partition defined by

$$
\lambda_{i}=w_{g+1-i}-g+i, \quad 1 \leq i \leq g,
$$

$s_{\mu}(t)$ is the Schur function corresponding to the partition $\mu$ and, in general, for two partitions $\mu=\left(\mu_{1}, \ldots, \mu_{l}\right)$ and $\nu=\left(\nu_{1}, \ldots, \nu_{l}\right), \mu \leq \nu$ if and only if $\mu_{i} \leq \nu_{i}$ for any $i$.

Define $b_{i j}, \widehat{q}_{i j}$ and $c_{i}$ by the expansions

$$
\begin{aligned}
& d u_{w_{i}}=\sum_{j=1}^{\infty} b_{i j} z^{j-1} d z, \quad \widehat{\omega}\left(p_{1}, p_{2}\right)=\left(\frac{1}{\left(z_{1}-z_{2}\right)^{2}}+\sum_{i, j=1}^{\infty} \widehat{q}_{i j} z_{1}^{i-1} z_{2}^{j-1}\right) d z_{1} d z_{2}, \\
& \log \left(z^{-g+1} \sqrt{\frac{d u_{2 g-1}}{d z}}\right)=\sum_{i=1}^{\infty} c_{i} \frac{z^{i}}{i}
\end{aligned}
$$

where $z_{i}=z\left(p_{i}\right) \cdot{ }^{1}$ Let us set

$$
B=\left(b_{i j}\right)_{1 \leq i \leq g, 1 \leq j}, \quad \widehat{q}(t)=\sum_{i, j=1}^{\infty} \widehat{q}_{i j} t_{i} t_{j}, \quad t={ }^{t}\left(t_{1}, t_{2}, \ldots\right) .
$$

Then we have

\section{Theorem 1.}

(i) There exists a constant $C$ such that

$$
\tau\left(t ; \xi^{A}\right)=C \exp \left(-\sum_{i=1}^{\infty} c_{i} t_{i}+\frac{1}{2} \widehat{q}(t)\right) \widehat{\sigma}(B t) .
$$

(ii) The tau function $\tau\left(t ; \xi^{A}\right)$ is a solution to the $a_{1}$-reduced KP-hierarchy.

Proof. The proof of this theorem is completely pararell to the case of $(n, s)$ curves in [20]. In fact the special property of $(n, s)$ curves we use in [20] is the existence of a holomorphic one form which vanishes at $\infty$ to the order $2 g-2$. In the present case $d u_{2 g-1}$ has such a property by Lemma 4 .

\footnotetext{
${ }^{1}$ In the right hand side of the defining equation of $c_{i}$ in [20], $z_{i}$ should be corrected as $z^{i} / i$.
} 
Combining the expansion (13) with Theorem 1 we have

Corollary 1. We have the following expansion

$$
C \widehat{\sigma}(u)=\left.s_{\lambda}(t)\right|_{t_{w_{i}}=u_{w_{i}}}+\cdots,
$$

where $\lambda$ is defined by (14) and $\cdots$ part is a series in $\prod_{i=1}^{g} u_{w_{i}} \gamma_{i}, \sum_{i=1}^{g} \gamma_{i} w_{i}>\sum_{i=1}^{g} \lambda_{i}$.

Definition 1. We define the sigma function by

$$
\sigma(u)=C \widehat{\sigma}(u) .
$$

It follows from this definition and Corollary 1 that the series expansion of $\sigma(u)$ at the origin begins from Schur function corresponding to the gap sequence at $\infty$. It is possible to give more precise properties of the expansion of $\sigma(u)$ which is similar to the case of $(n, s)$ curves (see Appendix A).

\section{Addition formulae}

Our result is that all properties of the sigma functions of $(n, s)$ curves given in Sections 4 and 5 of the paper [21] are valid, formally without any change, for sigma functions of telescopic curves. The strategy of the proofs of theorems in this section is explained in the next section.

In order to state the results precisely we need the prime function of a telescopic curve which was introduced in [19] for $(n, s)$ curves.

Let $E\left(p_{1}, p_{2}\right)$ be the prime form of a telescopic curve $X$. Since $d u_{2 g-1}$ has a zero of order $2 g-2$ at $\infty$ by Lemma 4 , it is possible to define, as in the case of $(n, s)$ curves, the prime function $\tilde{E}\left(p_{1}, p_{2}\right)$ by

$$
\tilde{E}\left(p_{1}, p_{2}\right)=-E\left(p_{1}, p_{2}\right) \prod_{i=1}^{2} \sqrt{d u_{2 g-1}\left(p_{i}\right)} \exp \left(\frac{1}{2} \int_{p_{1}}^{p_{2}} d \mathbf{u} \eta_{1} \omega_{1}^{-1} \int_{p_{1}}^{p_{2}} d \mathbf{u}\right) .
$$

It is a multi-valued analytic function on $X \times X$ which has similar properties to that for $(n, s)$ curves.

For a partition $\lambda=\left(\lambda_{1}, \ldots, \lambda_{l}\right)$ and $0 \leq k \leq l$ we set

$$
\begin{aligned}
& \left(w_{l}, \ldots, w_{1}\right)=\left(\lambda_{1}+l-1, \lambda_{2}+l-2, \ldots, \lambda_{l}\right), \\
& N_{\lambda, k}=\lambda_{k+1}+\cdots+\lambda_{l}, \quad N_{\lambda, 1}^{\prime}=\lambda_{2}+\cdots+\lambda_{l}-l+1, \\
& c_{\lambda, k}^{\prime}=\frac{N_{\lambda, k} !}{l-k} \prod_{i=1}^{l-k}\left(w_{j}-w_{i}\right), \quad \tilde{c}_{\lambda}=\frac{N_{\lambda, 1}^{\prime} !}{\prod_{i=1}^{l-1}\left(w_{i}-1\right) !} \prod_{i<j}^{l-1}\left(w_{j}-w_{i}\right),
\end{aligned}
$$

where $c_{\lambda, l}^{\prime}$ is considered to be 1 .

The following theorems give the properties of the sigma function restricted to the Abel-Jacobi image $W_{k}$ of the $k$-th symmetric products of $X$ for $k<g$.

Theorem 2. Let $1 \leq k \leq g$ and $p_{1}, \ldots, p_{k} \in X$. Then

(i) We have

$$
\partial_{u_{1}}^{N_{\lambda, k}} \sigma\left(\sum_{i=1}^{k} p_{i}\right)=c_{\lambda, k}^{\prime} S_{\left(\lambda_{1}, \ldots, \lambda_{k}\right)}\left(z_{1}, \ldots, z_{k}\right)+\cdots
$$


where $\cdots$ part is a series in $z_{1}, \ldots, z_{k}$ containing only terms proportional to $\prod_{i=1}^{k} z_{i}^{\gamma_{i}}$ with $\sum_{i=1}^{k} \gamma_{i}>\sum_{i=1}^{k} \lambda_{i}$

(ii) The following expansion in $z_{k}$ holds:

$$
\partial_{u_{1}}^{N_{\lambda, k}} \sigma\left(\sum_{i=1}^{k} p_{i}\right)=\frac{c_{\lambda, k}^{\prime}}{c_{\lambda, k-1}^{\prime}} \partial_{u_{1}}^{N_{\lambda, k-1}} \sigma\left(\sum_{i=1}^{k-1} p_{i}\right) z_{k}^{\lambda_{k}}+O\left(z_{k}^{\lambda_{k}+1}\right) .
$$

\section{Theorem 3.}

(i) If $n<N_{\lambda, 1}^{\prime}$ we have, for $p_{1}, p_{2} \in X$,

$$
\partial_{u_{1}}^{n} \sigma\left(p_{1}-p_{2}\right)=0
$$

(ii) The following expansion with respect to $z_{i}=z\left(p_{i}\right), i=1,2$ is valid:

$$
\partial_{u_{1}}^{N_{\lambda, 1}^{\prime}} \sigma\left(p_{1}-p_{2}\right)=(-1)^{g-1} \tilde{c}_{\lambda}\left(z_{1} z_{2}\right)^{g-1}\left(z_{1}-z_{2}\right)(1+\cdots),
$$

where $\cdots$ part is a series in $z_{1}, z_{2}$ which contains only terms proportional to $z_{1}^{i} z_{2}^{j}$ with $i+j>0$.

(iii) We have

$$
\partial_{u_{1}}^{N_{\lambda, 1}^{\prime}} \sigma\left(p_{1}-p_{2}\right)=(-1)^{g-1} \frac{\tilde{c}_{\lambda}}{c_{\lambda, 1}^{\prime}} \partial_{u_{1}}^{N_{\lambda, 1}} \sigma\left(p_{1}\right) z_{2}^{g-1}+O\left(z_{2}^{g}\right)
$$

The next theorem gives the expression of the prime function as a derivative of the sigma function.

Theorem 4. Let $\lambda=\left(\lambda_{1}, \ldots, \lambda_{g}\right)$ be the partition corresponding to the gap sequence at $\infty$ of an $\left(a_{1}, \ldots, a_{m}\right)$ curve $X$. Then

$$
\tilde{E}\left(p_{1}, p_{2}\right)=(-1)^{g-1} \tilde{c}_{\lambda}^{-1} \partial_{u_{1}}^{N_{\lambda, 1}^{\prime}} \sigma\left(p_{1}-p_{2}\right) .
$$

Corollary 2. For $p \in X$ we have

$$
\tilde{E}(\infty, p)=c_{\lambda, 1}^{\prime}{ }^{-1} \partial_{u_{1}}^{N_{\lambda, 1}} \sigma(p) .
$$

Finally the addition formulae of sigma functions for telescopic curves are given by

\section{Theorem 5.}

(i) For $n \geq g$ and $p_{i} \in X, 1 \leq i \leq n$,

$$
\frac{\sigma\left(\sum_{i=1}^{n} p_{i}\right) \prod_{i<j} \partial_{u_{1}}^{N_{\lambda, 1}^{\prime}} \sigma\left(p_{j}-p_{i}\right)}{\prod_{i=1}^{n}\left(\partial_{u_{1}}^{N_{\lambda, 1}} \sigma\left(p_{i}\right)\right)^{n}}=\tilde{b}_{\lambda, n} \operatorname{det}\left(\varphi_{i}\left(p_{j}\right)\right)_{1 \leq i, j \leq n},
$$

with

$$
\tilde{b}_{\lambda, n}=(-1)^{\frac{1}{2} g n(n-1)} \tilde{c}_{\lambda}^{\frac{1}{2} n(n-1)}\left(c_{\lambda, 1}^{\prime}\right)^{-n^{2}} .
$$


(ii) For $n<g$

$$
\frac{\partial_{u_{1}}^{N_{\lambda, n}} \sigma\left(\sum_{i=1}^{n} p_{i}\right) \prod_{i<j} \partial_{u_{1}}^{N_{\lambda, 1}^{\prime}} \sigma\left(p_{j}-p_{i}\right)}{\prod_{i=1}^{n}\left(\partial_{u_{1}}^{N_{\lambda, 1}} \sigma\left(p_{i}\right)\right)^{n}}=b_{\lambda, n}^{\prime} \operatorname{det}\left(\varphi_{i}\left(p_{j}\right)\right)_{1 \leq i, j \leq n},
$$

with

$$
b_{\lambda, n}^{\prime}=(-1)^{\frac{1}{2} g n(n-1)} \tilde{c}_{\lambda}^{\frac{1}{2} n(n-1)}\left(c_{\lambda, 1}^{\prime}\right)^{-n^{2}} c_{\lambda, n}^{\prime}
$$

The addition formulae of this kind were firstly derived by Ônishi [23] for the hyperelliptic sigma functions. We remark that the formulae in this theorem are written using algebraic data only, the sigma function, its derivatives and the algebraic functions $\varphi_{i}$. This fact makes it possible to study the restriction of the formulae on $W_{k}$ to lower strata $W_{k^{\prime}}$ for $k^{\prime}<k$ as in (ii). This is a main difference of our formulae and Fay's general addition formulae [9].

\section{Proofs}

In [21] properties of the sigma function of an $(n, s)$ curve have been proved by establishing the corresponding properties of Schur and tau functions. In this paper we exclusively consider $t_{1}$-derivatives and $u_{1}$-derivatives. We omit the results on " $a$-derivatives" in [21], since they are not used in addition formulae for general telescopic curves.

As far as $t_{1}$-derivatives are concerned, all the statements for Schur and tau functions in [21] hold, as stated there, for Schur function $s_{\lambda}(t)$ associated with any partition $\lambda$ and the tau functions $\tau(t)$ which have the expansion of the form

$$
\tau(t)=s_{\lambda}(t)+\sum_{\lambda<\mu} \xi_{\mu} s_{\mu}(t)
$$

Then Theorems 2, 3, 4, Corollary 2 can be proved in a similar manner to the case of $(n, s)$ curves using Theorem 1.

By Theorem 4 the prime function of a telescopic curve can be written as a derivative of the sigma function. Conversely the sigma function can be expressed by using the prime function and algebraic functions $\varphi_{i}$ as in the case of an $(n, s)$ curve.

Theorem 6. For $n \geq g$ and $p_{i} \in X, 1 \leq i \leq n$,

$$
\sigma\left(\sum_{i=1}^{n} p_{i}\right)=\frac{\prod_{i=1}^{n} \tilde{E}\left(\infty, p_{i}\right)^{n}}{\prod_{i<j}^{n} \tilde{E}\left(p_{i}, p_{j}\right)} \operatorname{det}\left(\varphi_{i}\left(p_{j}\right)\right)_{1 \leq i, j \leq n} .
$$

Expanding (15) in $z\left(p_{n}\right)$ successively with the help of Theorem 2 we get

Corollary 3. For $n<g$ we have

$$
\partial_{u_{1}}^{N_{\lambda, n}} \sigma\left(\sum_{i=1}^{n} p_{i}\right)=c_{\lambda, n}^{\prime} \frac{\prod_{i=1}^{n} \tilde{E}\left(\infty, p_{i}\right)^{n}}{\prod_{i<j}^{n} \tilde{E}\left(p_{i}, p_{j}\right)} \operatorname{det}\left(\varphi_{i}\left(p_{j}\right)\right)_{1 \leq i, j \leq n} .
$$

Theorem 5 can be obtained from Theorem 6 and Corollary 3 by substituting the sigma function expression of the prime function given by Theorem 4 and Corollary 2 . 


\section{Example: $(4,6,5)$-curve}

In this section we give an explicit example of the addition formulae in the case of a $(4,6,5)$ curve $X$. By Example 2(ii) in Section 2, the genus of $X$ is 4 . The gap sequence at $\infty$ is $\left(w_{1}, w_{2}, w_{3}, w_{4}\right)=(1,2,3,7)$. The partition corresponding to the gap sequence at $\infty$ is $\lambda=$ $\left(\lambda_{1}, \lambda_{2}, \lambda_{3}, \lambda_{4}\right)=(4,1,1,1)$. Therefore we have $N_{\lambda, 0}=7, N_{\lambda, 1}=3, N_{\lambda, 2}=2, N_{\lambda, 3}=1$, $N_{\lambda, 1}^{\prime}=0, c_{\lambda, 1}^{\prime}=c_{\lambda, 2}^{\prime}=c_{\lambda, 3}^{\prime}=\tilde{c}_{\lambda}=1$. On the other hand we have $\varphi_{1}=1, \varphi_{2}=x_{1}, \varphi_{3}=x_{3}$, $\varphi_{4}=x_{2}$. Therefore the addition formulae given in Theorem 5 for $n=2,3,4$ are as follows:

(i) For $n=2$,

$$
\frac{\left(\partial_{u_{1}}^{2} \sigma\left(p_{1}+p_{2}\right)\right) \sigma\left(p_{2}-p_{1}\right)}{\left(\partial_{u_{1}}^{3} \sigma\left(p_{1}\right)\right)^{2}\left(\partial_{u_{1}}^{3} \sigma\left(p_{2}\right)\right)^{2}}=x_{1}\left(p_{2}\right)-x_{1}\left(p_{1}\right) .
$$

(ii) For $n=3$,

$$
\frac{\partial_{u_{1}} \sigma\left(p_{1}+p_{2}+p_{3}\right) \prod_{1 \leq i<j \leq 3} \sigma\left(p_{j}-p_{i}\right)}{\prod_{j=1}^{3}\left(\partial_{u_{1}}^{3} \sigma\left(p_{j}\right)\right)^{3}}=\left|\begin{array}{ccc}
1 & 1 & 1 \\
x_{1}\left(p_{1}\right) & x_{1}\left(p_{2}\right) & x_{1}\left(p_{3}\right) \\
x_{3}\left(p_{1}\right) & x_{3}\left(p_{2}\right) & x_{3}\left(p_{3}\right)
\end{array}\right| .
$$

(iii) For $n=4$,

$$
\frac{\sigma\left(p_{1}+p_{2}+p_{3}+p_{4}\right) \prod_{1 \leq i<j \leq 4} \sigma\left(p_{j}-p_{i}\right)}{\prod_{j=1}^{4}\left(\partial_{u_{1}}^{3} \sigma\left(p_{j}\right)\right)^{4}}=\left|\begin{array}{cccc}
1 & 1 & 1 & 1 \\
x_{1}\left(p_{1}\right) & x_{1}\left(p_{2}\right) & x_{1}\left(p_{3}\right) & x_{1}\left(p_{4}\right) \\
x_{3}\left(p_{1}\right) & x_{3}\left(p_{2}\right) & x_{3}\left(p_{3}\right) & x_{3}\left(p_{4}\right) \\
x_{2}\left(p_{1}\right) & x_{2}\left(p_{2}\right) & x_{2}\left(p_{3}\right) & x_{2}\left(p_{4}\right)
\end{array}\right| .
$$

\section{A Series expansion of sigma function}

Using Theorem 6 in a similar manner to the case of $(n, s)$ curves (cf. [19]), we can show the following theorem.

Theorem 7. The expansion of $\sigma(u)$ at the origin takes the form

$$
\sigma(u)=\left.s_{\lambda}(t)\right|_{t_{w_{i}}=u_{w_{i}}}+\sum_{\sum_{i=1}^{g} \gamma_{i} w_{i}>\sum_{i=1}^{g} \lambda_{i}} \tilde{e}_{\gamma_{1} \ldots \gamma_{g}} u_{w_{1}}^{\gamma_{1}} \cdots u_{w_{g}}^{\gamma_{g}}
$$

where $\tilde{e}_{\gamma_{1} \ldots \gamma_{g}}$ belongs to $\mathbb{Q}\left[\left\{\kappa_{j_{1} \ldots j_{m}}^{(i)}\right\}\right]$ and is homogeneous of degree $\sum_{i=1}^{g} \gamma_{i} w_{i}-\sum_{i=1}^{g} \lambda_{i}$ if $\tilde{e}_{\gamma_{1} \ldots \gamma_{g}} \neq 0$.

Remark 1. It is possible to prove the above theorem using the relation with the tau function as in [20].

\section{Acknowledgements}

The authors would like to thank the referees for the useful comments. This research was partially supported by Grant-in-Aid for JSPS Fellows (22-2421) and for Scientific Research (C) 23540245 from Japan Society for the Promotion of Science. 


\section{References}

[1] Ayano T., Sigma functions for telescopic curves, Osaka J. Math., to appear, arXiv:1201.0644.

[2] Baker H.F., Abelian functions. Abel's theorem and the allied theory of theta functions, Cambridge Mathematical Library, Cambridge University Press, Cambridge, 1995.

[3] Braden H.W., Enolski V.Z., Fedorov Yu.N., Dynamics on strata of trigonal Jacobians and some integrable problems of rigid body motion, arXiv:1210.3596.

[4] Brauer A., On a problem of partitions, Amer. J. Math. 64 (1942), 299-312.

[5] Brauer A., Seelbinder B.M., On a problem of partitions. II, Amer. J. Math. 76 (1954), 343-346.

[6] Buchstaber V.M., Enolski V.Z., Leykin D.V., Multi-dimensional sigma functions, arXiv:1208.0990.

[7] Bukhshtaber V.M., Enolski V.Z., Leykin D.V., Rational analogues of abelian functions, Funct. Anal. Appl. 33 (1999), 83-94.

[8] Eilbeck J.C., Enolski V.Z., Gibbons J., Sigma, tau and Abelian functions of algebraic curves, J. Phys. A: Math. Theor. 43 (2010), 455216, 20 pages, arXiv:1006.5219.

[9] Fay J.D., Theta functions on Riemann surfaces, Lecture Notes in Mathematics, Vol. 352, Springer-Verlag, Berlin, 1973.

[10] Harnad J., Enolski V.Z., Schur function expansions of KP $\tau$-functions associated to algebraic curves, Russ. Math. Surv. 66 (2011), 767-807, arXiv:1012.3152.

[11] Kirfel C., Pellikaan R., The minimum distance of codes in an array coming from telescopic semigroups, IEEE Trans. Inform. Theory 41 (1995), 1720-1732.

[12] Klein F., Ueber hyperelliptische Sigmafunctionen, Math. Ann. 27 (1886), 431-464.

[13] Klein F., Ueber hyperelliptische Sigmafunctionen (Zweite Abhandlung), Math. Ann. 32 (1888), 351-380.

[14] Komeda J., Matsutani S., Previato E., The sigma function for Weierstrass semigroups $\langle 3,7,8\rangle$ and $\langle 6,13,14,15,16\rangle$, arXiv:1303.0451.

[15] Korotkin D., Shramchenko V., On higher genus Weierstrass sigma-function, Phys. D 241 (2012), 2086-2094, arXiv:1201.3961.

[16] Matsutani S., Sigma functions for a space curve $(3,4,5)$ type with an appendix by J. Komeda, arXiv:1112.4137.

[17] Micale V., Olteanu A., On the Betti numbers of some semigroup rings, Matematiche (Catania) 67 (2012), 145-159, arXiv:1111.1433.

[18] Miura S., Linear codes on affine algebraic curves, Trans. IEICE J81-A (1998), 1398-1421.

[19] Nakayashiki A., On algebraic expressions of sigma functions for (n,s) curves, Asian J. Math. 14 (2010), 175-211, arXiv:0803.2083.

[20] Nakayashiki A., Sigma function as a tau function, Int. Math. Res. Not. 2010 (2010), no. 3, 373-394, arXiv:0904.0846.

[21] Nakayashiki A., Yori K., Derivatives of Schur, tau and sigma functions on Abel-Jacobi images, in Symmetries, Integrable Systems and Representations, Springer Proceedings in Mathematics 83 Statistics, Vol. 40, Editors K. Iohara, S. Morier-Genoud, B. Remy, Springer-Verlag, London, 2013, 429-462, arXiv:1205.6897.

[22] Nishijima D., Order counting algorithm for Fermat curves and Klein curves using $p$-adic cohomology, Master's Thesis, Osaka University, 2008.

[23] Ônishi Y., Determinant expressions for hyperelliptic functions (with an Appendix by Shigeki Matsutani), Proc. Edinb. Math. Soc. (2) 48 (2005), 705-742, math.NT/0105189.

[24] Sato M., Sato Y., Soliton equations as dynamical systems on infinite-dimensional Grassmann manifold, in Nonlinear Partial Differential Equations in Applied Science (Tokyo, 1982), North-Holland Math. Stud., Vol. 81, Editors P.D. Lax, H. Fujita, G. Strang, North-Holland, Amsterdam, 1983, 259-271. 